\title{
Sustainment of the Reversed Shear Magnetic Field Configuration with Near-Rational q-min Value with the Help of the Rotating Magnetic Field
}

\author{
S. Kasilov*1, M. F. Heyn ${ }^{2}$, I. B. Ivanov ${ }^{3}$, and W. Kernbichler ${ }^{2}$ \\ ${ }^{1}$ Institute of Plasma Physics, National Science Center "Kharkov Institute of Physics and Technology", ul. \\ Akademicheskaya 1, 61108 Kharkov, Ukraine \\ 2 Institut für Theoretische Physik - Computational Physics, Technische Universität Graz, Petersgasse 16, A- \\ 8010 Graz, Austria \\ ${ }^{3}$ Petersburg Nuclear Physics Institute, 188300, Gatchina, Leningrad Region, Russia
}

Received 19 October 2005, accepted 9 February 2006

Published online 22 August 2006

Key words Tokamak, reversed shear configuration, rotating magnetic fields. PACS 52.55.Fa

It is proposed to use rotating magnetic fields for automatic control of the $q$-profile of a reversed magnetic shear configuration in order to sustain its $q$ minimum value in the vicinity of a low order rational number.

(c) 2006 WILEY-VCH Verlag GmbH \& Co. KGaA, Weinheim

\section{Introduction}

Tokamak configurations with reversed magnetic shear are used for the creation of internal transport barriers. Due to the low variation of $q$ with radius near the shear reversal point ( $q$-minimum point), the distance between the neighboring resonant magnetic surfaces is increased which may prevent overlapping of resonant turbulent modes with different helicities and may lead to the local reduction of transport. It has been observed experimentally [1] and also found in global turbulence modelling [2] that when the $q$-minimum value, $q_{\min }$, is close to a low order rational number, the reduction of anomalous transport becomes stronger. This is due to the fact that there are no other low order rational numbers in a certain vicinity of a given low order rational number as shown in Fig. 1 (see, e.g., $[2,3]$ ).

Although it seems attractive, it is technically difficult to sustain such a configuration where $q_{\min }$ never gets below a low order rational number. If this happens, MHD modes are excited which leads to a severe confinement degradation [1].

One of the main methods for creation of the reversed shear configurations is the off-axis co-ECCD which creates the hollow profile of the total current. This method allows rather accurate control of the current profile, however, it is not sensitive to the $q$-profile. Here, the possibility is studied to use a small additional current drive from the rotating magnetic field in order to control the current near the low order rational $q$-value. At low frequencies rotating fields interact resonantly with plasma in the vicinity of local Alfvén resonances, $k_{\|}=$ $(m+n q) /(q R)= \pm \omega / v_{A}$, which can appear near rational $q$ value where $m+n q$ is small enough. Here, $k_{\|}, m$, $n, q, R, \omega, v_{A}$ are parallel wave vector, poloidal and toroidal wave numbers, safety factor, major radius, rotation frequency, and Alfvén velocity, respectively. If only one out of two resonances is realized (see Fig. 2), the net parallel momentum is coupled to the electrons which leads to current generation. This current for a counter rotating field compensates partly the ECCD current and thus might prevent the further reduction of $q$ below the resonant value $q=m / n$.

${ }^{*}$ Corresponding author: e-mail: kasilov @itp.tugraz.at 


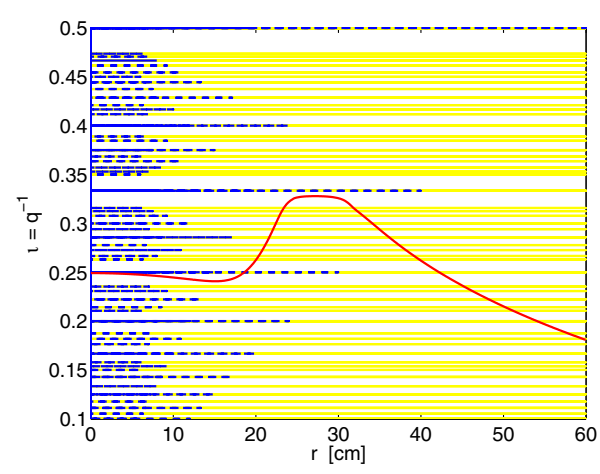

Fig. 1 Profile of the rotational transform angle, $\iota(r)=$ $1 / q(r)$ (red) and the resonant values of $\iota$ for the perturbations with limited spectrum over poloidal wave-numbers, $-M<m<M$ (yellow). Length of blue lines scales inversely with $m$. (Online colour: www.cpp-journal.org).

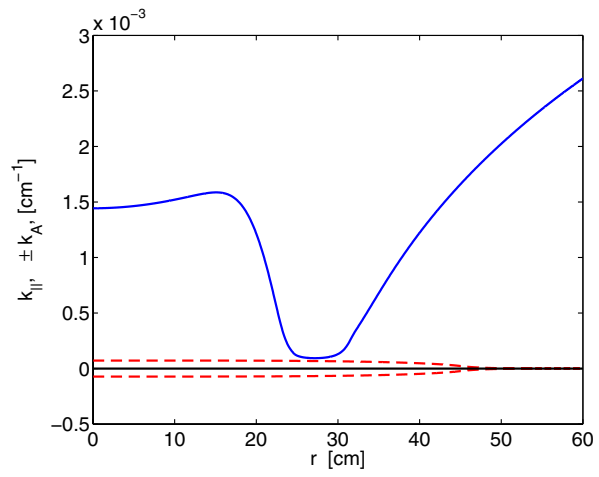

Fig. 2 Profiles of the parallel wave-vector $k_{\|}$(blue) and the Alfvén wave-vector $k_{A}= \pm \omega / v_{A}$ (red). (Online colour: www.cpp-journal.org).

\section{The Model}

The possibility of a dynamic equilibrium has been looked for by solving numerically the poloidal magnetic field diffusion equation in cylindrical geometry,

$$
\frac{\partial B_{0 \theta}}{\partial t}=\frac{\partial}{\partial r} D_{s}\left(\frac{1}{r} \frac{\partial}{\partial r} r B_{0 \theta}-\frac{4 \pi}{c} j_{t o t}\right),
$$

where $D_{s}=c^{2} /\left(4 \pi \sigma_{\|}\right)$is the magnetic field diffusion coefficient, $\sigma_{\|}$is a parallel plasma conductivity, and $j_{t o t}=j_{O}+j_{E C C D}+j_{D E D}$ is the toroidal current density with $j_{O}, j_{E C C D}$ and $j_{D E D}$ being the inductive, the EC driven and the DED driven toroidal current densities in case of a steady state. Currents $j_{O}, j_{E C C D}$ are assumed constant whereas $j_{D E D}$ is updated each time step from the numerical solution of Maxwell equations for the perturbation field. One should notice that the direction of the ECCD current and the direction of the DED driven current can be controlled by launch angle and direction of the rotation of the DED magnetic field, respectively. Therefore, a proper sign of the additional current can always be achieved.

Maxwell equations for the perturbation field

$$
\nabla \times \mathbf{E}=\frac{i \omega}{c} \mathbf{B}, \quad \nabla \times \mathbf{B}=-\frac{i \omega}{c} \hat{\varepsilon} \cdot \mathbf{E},
$$

are solved for the geometry of the inhomogeneous plasma cylinder with a rotational transform of the magnetic field. A strongly simplified homogeneous plasma model for the dielectric permittivity tensor $\hat{\varepsilon}$ is used which in the coordinate frame where the $z$-axis is parallel to the unperturbed magnetic field $\mathbf{B}_{0}$ is of the form

$$
\hat{\varepsilon}=\left(\begin{array}{ccc}
\varepsilon_{1} & i \varepsilon_{2} & 0 \\
-i \varepsilon_{2} & \varepsilon_{1} & 0 \\
0 & 0 & \varepsilon_{3}
\end{array}\right),
$$

where

$$
\begin{aligned}
& \varepsilon_{1}=1+\frac{\omega_{p i}^{2}}{\omega_{c i}^{2}-\omega^{2}}, \quad \varepsilon_{2}=\frac{\omega}{\omega_{c i}} \frac{\omega_{p i}^{2}}{\omega_{c i}^{2}-\omega^{2}}, \\
& \varepsilon_{3}=1+\sum_{\alpha=e, i} \frac{\omega_{p \alpha}^{2}}{k_{\|}^{2} v_{T \alpha}^{2}}\left(1+i \sqrt{\pi} Z_{\alpha} w\left(Z_{\alpha}\right)\right),
\end{aligned}
$$

with $Z_{\alpha}=\omega /\left(\sqrt{2} k_{\|} v_{T \alpha}\right)$ and the ion cyclotron frequency $\omega_{c i}=e B_{0} /\left(m_{i} c\right)$. This model model for the plasma conductivity is not consistent with the equilibrium plasma current, pressure and rotation velocity which 
are important at low frequencies but it is simple and, therefore, fast enough to follow the time evolution of the poloidal magnetic field. The DED driven current density is computed from the electron power absorption density using the local current drive efficiency $\eta$ [4] taking into account toroidal trapping of resonant electrons in the following way $j_{D E D}(r)=\eta\left(r, k_{\|}\right) p_{a b s}(r)$. Typically, this efficiency is low, $\eta \sim 0.03 \mathrm{~A} / \mathrm{W}$.

\section{Results}

A single mode $m=3, n=1$ excited by the external coil is considered. The plasma density and the temperature profiles are shown in Fig. 3. The other parameters are: main magnetic field $B_{0}=20 \mathrm{kG}$, major radius $R_{0}=175$ $\mathrm{cm}$, coil radius $r_{a}=53 \mathrm{~cm}$, wall radius $r_{w}=60 \mathrm{~cm}$, perturbation frequency $\omega / 2 \pi=10 \mathrm{kHz}$. In case of this simple model for the plasma response, a steady equilibrium state had been reached with an ECCD current $I_{E C C D}=88 \mathrm{kA}$ and a DED driven current $I_{D E D}=27 \mathrm{kA}$ (see Fig. 4) and the desired equilibrium with $q-\min$ slightly above $q=3$ had been achieved. However, since the plasma response model used in the present paper

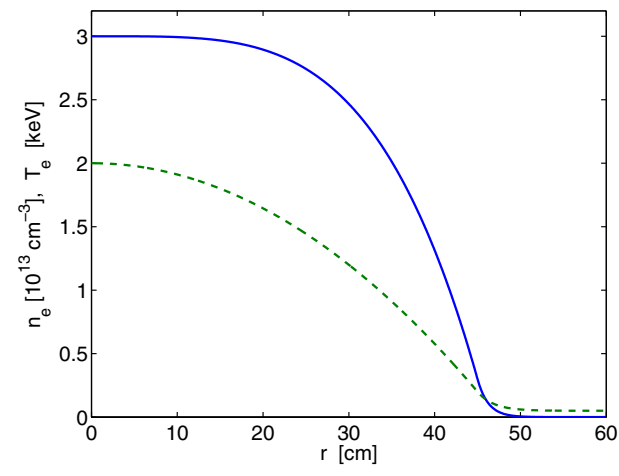

Fig. 3 Electron density (blue) and temperature (green) profiles. (Online colour: www.cpp-journal.org).

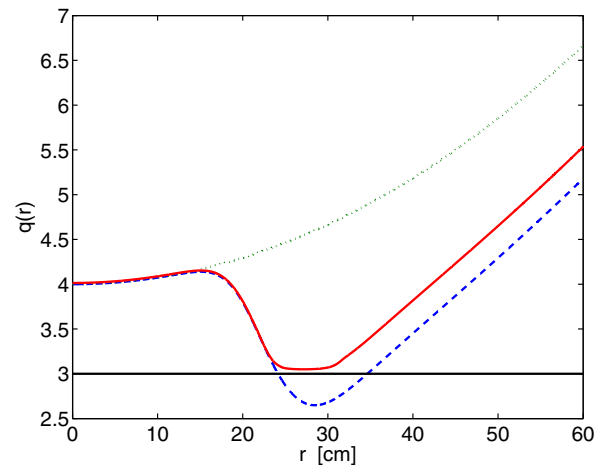

Fig. 4 Safety factor profiles. Green - inductive current drive, blue - inductive and EC current drive, red - inductive, EC and DED current drive. (Online colour: www.cpp-journal.org).

is not consistent with the equilibrium, the possibility of such a dynamic equilibrium has still to be investigated using a plasma response model consistent with the equilibrium plasma parameters [5] and taking into account also the effect of the perturbation field on the plasma rotation. The latter effect can significantly reduce the power absorption from the perturbation field if the electron fluid velocity approaches the perturbation field rotation velocity.

At the same time, the mechanism of the feedback current generation should not depend on the concrete model used. It uses the fact that the interaction of the rotating field with the plasma takes place near Alfven resonances where the parallel phase velocity of the field equals the local Alfven velocity. The sign of the phase velocity at a particular Alfven resonance depends on the sign of the difference $q-q_{r}$ where $q_{r}=m / n$ is a rational number. If this difference has the same sign everywhere as it is the case in the present configuration, the phase velocity has a definite sign everywhere and this, in turn, leads to a net current generation in one direction. Therefore, a more realistic model should not remove the effect completely, but it might predict different parameters of the rotating field which eventually are not feasible for TEXTOR DED any more.

Acknowledgements This work has been carried out within the Association EURATOM-ÖAW and with funding from the Austrian Science Fund (FWF) under contract P15956-N08 and P16157-N08.

\section{References}

[1] K. A. Razumova, V. V. Alikaev, A. A. Borschegovskii, et al, Plasma Phys. Contr. Fusion 42, 973 (2000).

[2] X. Garbet, C. Bourdelle, G. T. Hoang, et al, Phys. Plasmas 8, 2793 (2001).

[3] S. V. Kasilov, D. Reiter, A. M. Runov, et al, Plasma Phys. Contr. Fusion 44, 985 (2002).

[4] Ya. I. Kolesnichenko, V. V. Parail, G. V. Pereverzev, in Reviews of Plasma Physics Vol.17, edited by B.B. Kadomtsev, (Consultants Bureau, New Yourk, 1992), p. 1.

[5] M. F. Heyn, I. B. Ivanov, S. V. Kasilov, W. Kernbichler, Nucl. Fusion 46, 159-169 (2006). 